\title{
IMAGING OF ELECTRODE MOVEMENT AND CONDUCTIVITY CHANGE IN ELECTRICAL IMPEDANCE TOMOGRAPHY
}

\author{
Camille Gómez-Laberge \\ School of Information Technology \\ and Engineering (SITE), \\ University of Ottawa, Canada \\ e-mail: cgomez@site.uottawa.ca
}

\author{
Andy Adler \\ School of Information Technology \\ and Engineering (SITE), \\ University of Ottawa, Canada \\ e-mail: adler@site.uottawa.ca
}

\begin{abstract}
Electrical Impedance Tomography (EIT) applies and measures electrical energy on the boundary of a medium to produce an image of its internal impedance distribution. In many medical applications, such as imaging of the chest, the electrodes move during measurements, introducting artefacts into the calculated images. This paper proposes a new algorithm that compensates for electrode movement during difference EIT measurements. The reconstructed image is calculated by regularizing the image reconstruction matrix based on the sensitivity and smoothness of the conductivity and movement data.

A comparison of the standard and proposed EIT reconstruction methods is made. Images are reconstructed from $2 D$ and $3 D$ simulations exhibiting conductivity changes and electrode movements. Results show the proposed method yields a good estimation of electrode movement and a significant improvement in conductivity image reconstructions.
\end{abstract}

Keywords - Electrical impedance tomography; inverse problems; regularization.

\section{Introduction}

Electrical Impedance Tomography (EIT) applies and measures electrical energy on the boundary of a medium to produce an image of its internal impedance distribution. In many medical applications, such as imaging of the chest, the boundary shape changes during measurements, which results in movement of the electrodes, and causes significant deterioration in the reconstructed image quality. Movements greater than $1 \%$ of the medium diameter cause severe artefacts in simulated image reconstructions [5]. This is particularly the case in medical imaging where body movements due to breathing and posture introduce uncertainty in electrode position relative to the organs of interest. For example, when imaging breathing, boundary movements of $10 \%$ of the medium diameter can be observed. The effect of boundary motion and electrode position uncertainty in EIT is also discussed throughout the literature $[2],[3],[4],[6],[7]$, and [8].

This study proposes a new method to reconstruct the change in internal conductivity distribution and electrode movement between measurements. The second section describes the EIT inverse problem and develops the regularization terms used in the reconstruction algorithm. The third section compares the image reconstruction of both the standard and proposed methods and analyzes the obtained results. Finally, a concluding section discusses the relevant applications that benefit from this study.

\section{Methods}

This section develops the forward and inverse solutions for difference EIT. The augmented Jacobian is formulated in the forward solution section and the a priori reconstruction matrices are formulated in the inverse solution section. A brief definition of our figure-of-merit for quantifying the effect of artefacts introduced by the inverse solution is also given.

The medium under study is represented by a finite element model (FEM) partitioned into $n_{N}$ elements of triangular or tetrahedral shape. $n_{E}$ Electrodes are defined on the medium boundary, using either point or complete electrode models.

\section{$2.1 \quad$ Difference EIT}

Difference EIT data is obtained by periodically applying a current injection and voltage measurement protocol, where each period is referred to as a measurement frame. Each frame collects a vector of measurements

$$
\mathbf{z}=\mathbf{v}_{t_{2}}-\mathbf{v}_{t_{1}}=\Delta \mathbf{v}
$$

between measurements at taken at times $t_{1}$ and $t_{2}$.

During this time interval the medium undergoes a change in internal conductivity distribution $\Delta \sigma$ and a change in boundary shape. $\Delta \sigma$ is represented by a vector $n_{N} \times 1$ of the change in conductivity in each finite element, $\Delta \sigma=$ $\sigma_{t_{2}}-\sigma_{t_{1}}$, while boundary shape change is represented by the vector displacement of each electrode. All nodes associated with an electrode are assumed to be subject to the same displacement, and thus boundary shape change is represented by a vector of size $n_{D} n_{E} \times 1$, where $n_{D}$ is the model dimention $(2 \mathrm{D}$ or $3 \mathrm{D})$. The reconstructed image, denoted as $\hat{x}$, reflects both the conductivity change and boundary movement, and is of size $\left(n_{N}+n_{D} n_{E}\right) \times 1$.

\subsection{Forward solution}

The forward calculations produce the potential difference measurements from the conductivity change and electrode movement data relative to the medium with a homogeneous conductivity distribution $\sigma_{h}$.

$$
\mathbf{z}=\left.F(\mathbf{x})\right|_{\sigma_{h}}
$$

The forward solution is implemented as the linearized model $\mathbf{z}=\mathbf{J} \mathbf{x}+\mathbf{n}$ where $\mathbf{J}$ is the Jacobian that quantifies the 
sensitivity of the forward operator in (2). Each element of $\mathbf{J}$ is calculated by small perturbations of each finite element in the forward solution such that

$$
\mathbf{J}_{i, j}=\frac{F_{i}\left(\mathbf{x}_{j}+\Delta \mathbf{x}_{j}\right)}{\Delta \mathbf{x}_{j}}
$$

Estimating $\mathbf{x}$ from (2) cannot be done directly with a least-squares solution since $\mathbf{J}$ has a condition number in the order of $10^{19}$ in our calculations, corresponding to a severely ill-posed problem. Thus, a direct inversion of (2) produces useless results since boundary measurements cannot determine the interior conductivity distribution uniquely.

\subsection{Inverse solution}

The image is estimated by a maximum a posteriori (MAP) regularization technique [1] that conditions the solution with Gaussian a priori estimates of the noise and image correlation matrices $\boldsymbol{\Sigma}_{n}$ and $\boldsymbol{\Sigma}_{x}$, respectively. The image estimate $\hat{\mathbf{x}}$ is given by the minimization problem,

$$
\hat{\mathbf{x}}=\underset{\mathbf{x}}{\arg \min }(\mathbf{z}-F(\mathbf{x}))^{t} \boldsymbol{\Sigma}_{n}^{-1}(\mathbf{z}-F(\mathbf{x}))+\mathbf{x}^{t} \boldsymbol{\Sigma}_{x}^{-1} \mathbf{x}
$$

where the expected conductivity of image elements and electrode movements are equiprobable in the positive or negative direction.

In our implementation of (4), the noise and image correlation matrices are represented by $\mathbf{W}$ and $\mathbf{R}$, respectively. We segregate the image components such that $\mathbf{R}$ is written in terms of conductivity $\mathbf{R}_{c}$, and movement $\mathbf{R}_{m}$, correlations. Thus our priors are defined as

$$
\boldsymbol{\Sigma}_{n}^{-1}=\frac{1}{\sigma_{n}^{2}} \mathbf{W}, \quad \boldsymbol{\Sigma}_{x}^{-1}=\frac{1}{\sigma_{c}^{2}} \mathbf{R}_{c}+\frac{1}{\sigma_{m}^{2}} \mathbf{R}_{m}
$$

where the $\sigma$-variables represent the a priori amplitude in the noise $\sigma_{n}^{2}$, conductivity $\sigma_{c}^{2}$, and movement data $\sigma_{m}^{2}$.

The $\mathbf{W}$ matrix is constructed assuming equal, additive Gaussian noise on each electrode channel. The $\mathbf{R}$ matrix is partitioned such that the upper $n_{N} \times n_{N}$ part, $1 / \sigma_{c}^{2} \mathbf{R}_{c}$, represents the covariance between conductivity changes of elements and the lower $n_{D} n_{E} \times n_{D} n_{E}$ part, $1 / \sigma_{m}^{2} \mathbf{R}_{m}$, represents the covariance between electrode movements. The conductivity changes are modelled with a smoothness constraint between adjacent elements such that $\mathbf{R}_{c}$ is a discrete Laplacian (spatial high pass) filter. A similar smoothness constraint is applied to electrode movement such that the movements of neighbouring electrodes are correlated since they are attached to the same region of the boundary.

It is necessary to introduce a parameter to normalize the numerical variation between components of $\mathbf{R}$ since they are of different unit. We define $\mu=\sigma_{c} / \sigma_{m}$ as the movement hyperparameter representing the reconstruction fidelity between conductivity and movement data. The movement partition $\mathbf{R}_{m}$ of the image prior matrix is scaled by $\mu^{2}$ such that the elements of $\mathbf{R}$ are given by

$$
\mathbf{R}_{i, j}= \begin{cases}n_{D}+1 & \text { if } i=j \text { and } i \leq n_{N} \\ -1 & \text { if } \operatorname{adj}(i, j) \text { and } i \leq n_{N} \\ 2.1 \mu^{2} & \text { if } i=j \text { and } i>n_{N} \\ -\mu^{2} & \text { if } \operatorname{adj}(i, j) \text { and } i>n_{N} \\ 0 & \text { otherwise }\end{cases}
$$

where $\operatorname{adj}(i, j)$ indicates that elements $i$ and $j$ represent adjacent elements or electrodes in the FEM.

The MAP estimate is implemented by applying the Jacobian in (3) and the priors from (5) to the expression to be minimized in (4). This yields the regularized linear inverse solution for the conductivity and movement $\hat{\mathbf{x}}$ based on the measured data $\mathbf{z}$.

$$
\hat{\mathbf{x}}=\left(\mathbf{J}^{t} \frac{1}{\sigma_{n}^{2}} \mathbf{W} \mathbf{J}+\frac{1}{\sigma_{c}^{2}} \mathbf{R}_{c}+\frac{1}{\sigma_{m}^{2}} \mathbf{R}_{m}\right)^{-1} \mathbf{J}^{t} \frac{1}{\sigma_{n}^{2}} \mathbf{W} \mathbf{z}
$$

This can be written in terms of the global hyperparameter $\lambda^{2}=\sigma_{n}^{2} / \sigma_{m}^{2}$ and the movement hyperparameter $\mu^{2}=$ $\sigma_{c}^{2} / \sigma_{m}^{2}$ where $\mathbf{R}=\mathbf{R}_{c}+\mu^{2} \mathbf{R}_{m}$. Since we assume the noise over each channel has equal variance $\sigma_{n}^{2}$, we have $\mathbf{W}=\mathbf{I}$. Thus from (7) we have the inverse solution conditionned by the regularization matrices $\mathbf{W}, \mathbf{R}_{c}$ and $\mathbf{R}_{m}$ and the hyperparameters $\lambda$ and $\mu$,

$$
\begin{aligned}
\hat{\mathbf{x}} & =\left(\mathbf{J}^{t} \mathbf{J}+\lambda^{2}\left(\mathbf{R}_{c}+\mu^{2} \mathbf{R}_{m}\right)\right)^{-1} \mathbf{J}^{t} \mathbf{z} \\
& =\left(\mathbf{J}^{t} \mathbf{J}+\lambda^{2} \mathbf{R}\right)^{-1} \mathbf{J}^{t} \mathbf{z} .
\end{aligned}
$$

\subsection{Artefact measurement}

The effect of reconstruction artefacts was found to be reduced in the proposed algorithm due to compensation for incorrect electrode positions. To quantify this effect, we define the artefact amplitude measure (AAM) as follows: A reconstruction artefact is defined to be an element reconstructed with a non-zero conductivity change that corresponds to simulated elements without any conductivity changes. AAM, is defined to be

$$
\mathrm{AAM}=\sqrt{\frac{\sum_{i \in L} A_{i} \mathbf{x}_{i}^{2}}{\sum_{i \in L} A_{i}}}
$$

where $A_{i}$ is the area (in 2D) or volume (in 3D) of each element, and $L$ is the set of elements which ought to be conductivity change free. Thus, $L$ includes all elements which do not overlap with any contrast element in the forward model.

\section{Results}

Simulated data were generated using the EIDORS open-source tomographic image reconstruction software suite.Image reconstructions for $2 \mathrm{D}$ and $3 \mathrm{D}$ models were compared using the standard and the proposed algorithms. The 2D simulations use a circular (unit-radius) forward 


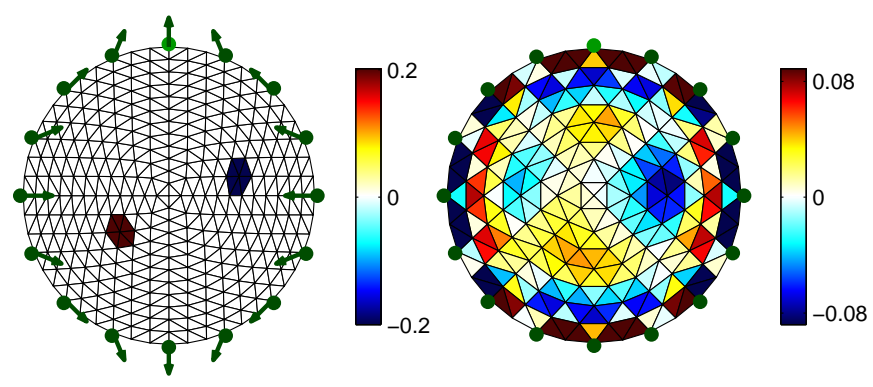

Figure 1. 2D simulation with boundary movement and standard method reconstruction. Arrows indicate each electrode's movement, and are scaled by $20 \times$. Left: Simulated 576 element FEM with conductivity change and electrode movement from an elliptical deformation of $1 \%$ of the medium diameter. Additive noise yielding a $20 \mathrm{~dB}$ SNR corrupts the simulation data. Right: Standard method reconstruction using a 256 element FEM with $\lambda=10^{-2}$ (Artefact amplitude $\mathrm{AAM}=0.0616)$.

model of 576 triangular elements and 313 nodes. The difference EIT data was simulated by calculating $\mathbf{v}_{t_{1}}$ for a homogeneous conductivity $\sigma_{h}$ throughout the medium and calculating $\mathbf{v}_{t_{2}}$ for two small inhomogeneities, $0.8 \times \sigma_{h}$ shown in blue and $1.2 \times \sigma_{h}$ shown in red in the left part of figure 1 . Boundary movement was simulated by displacing the mesh nodes in an elliptical deformation such that the boundary nodes sustained a $1 \%$ elongation along the $y$-axis and a $1 \%$ compression along the $x$-axis of the medium diameter. Noise was added in the simulation such that the SNR was $20 \mathrm{~dB}$, where the signal was defined to be the measurement power, $\|\mathbf{z}\|^{2}$.

The standard reconstruction, shown on the right part of figure 1, does not account for electrode movement and consequently shows severe artefacts along the boundary. The inhomogeneities also appear to have been displaced and show a reflected version opposite the centre of the medium.

The proposed method reconstructs the simulation for the same global hyperparameter value as the standard method, $\lambda=10^{-2}$, and two different values of the movement hyperparameter $\mu$. Figure 2 shows the proposed method reconstructions for $\mu=1$ on the left and $\mu=20$ on the right.

As stated above, the conductivity variation of the $2 \mathrm{D}$ simulated data was $0.4 \times \sigma_{h}$ and movements were on the order of $1 \%$ of the medium diameter. However, solving with $\mu=1$ implies that movements on the order of $40 \%$ are equivalent to a conductivity change of $\sigma_{c}$, since $\sigma_{m}=\sigma_{c} / \mu$. Thus, we observed an under-regularization of the movement calculations, resulting in somewhat sporadic movement vectors shown left in figure 2 . Solving with $\mu=20$ implies movements on the order of $2 \%$ are equivalent to conductivity changes of $\sigma_{c}$. This corresponds closely to the movements simulated and an adequate reconstruction of the electrode

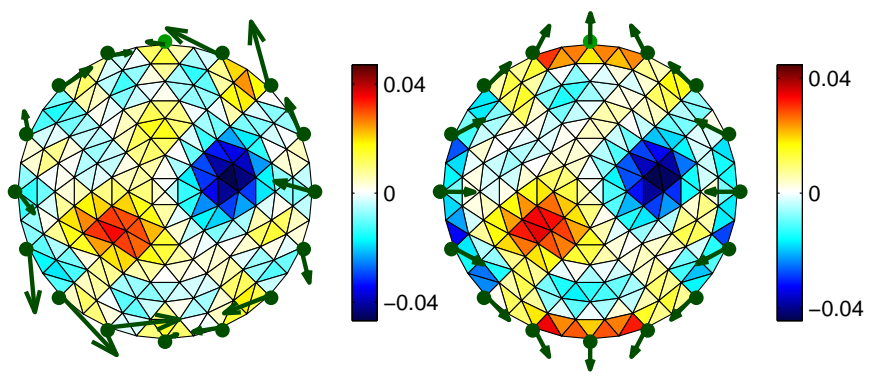

Figure 2. Proposed method reconstructions of the 2D simulation in figure 1. Arrows indicate each electrode's movement, and are scaled by $20 \times$. Left: Proposed method reconstruction using a 256 element FEM with $\lambda=10^{-2}$ and $\mu=1$ (AAM $\left.=0.0116\right)$. Right: Proposed method reconstruction using a 256 element FEM with $\lambda=10^{-2}$ and $\mu=20$ (AAM $=$ $0.0135)$.

movements is observed right in figure 2. It should be noted however that both values of $\mu$ provide movement compensation, illustrating an observable reduction in artefacts resulting in a $78 \%$ smaller AAM for $\mu=20$ and $81 \%$ smaller AAM for $\mu=1$, compared to the standard method.

The 3D simulations use a cylindrical (unit-radius and of 3 units height) forward model of 828 tetrahedral elements and 252 nodes. The difference EIT data was simulated by calculating $\mathbf{v}_{t_{1}}$ for a homogeneous conductivity $\sigma_{h}$ throughout the medium and calculating $\mathbf{v}_{t_{2}}$ for two inhomogeneities, $0.80 \times \sigma_{h}$ in blue and $1.15 \times \sigma_{h}$ in red, on three horizontal slices of the medium, shown in the left column of figure 3. Boundary movement was simulated by displacing the mesh nodes in a complex elliptical deformation such that the boundary nodes sustained a $1 \%$ elongation along the $x$-axis and a $1 \%$ compression along the $y$-axis at the $z=0$ plane and with same deformation at the $z=3$ plane except with $x$ and $y$ axes interchanged. The arrows showing movement are scaled by $10 \times$. Noise was added in the same way as the $2 \mathrm{D}$ simulation such that the SNR was $20 \mathrm{~dB}$.

A reduction of the artefacts on the boundary is observable in the proposed method and the AAM is $73 \%$ smaller for $\mu=20$, compared to the standard method. The proposed method was also capable of calculating reasonable electrode movements.

\section{Conclusions}

It is well known that boundary movement in flexible media EIT causes a severe reduction in image quality due to electrode position uncertainty. Physiological imaging applications such as pulmonary, gastrointestinal and mammographic EIT suffer from this effect due to inevitable subject breathing and slight posture changes. Our proposed algorithm dramatically reduces movement artefacts by regularizing the reconstructed image based on both, conduc- 


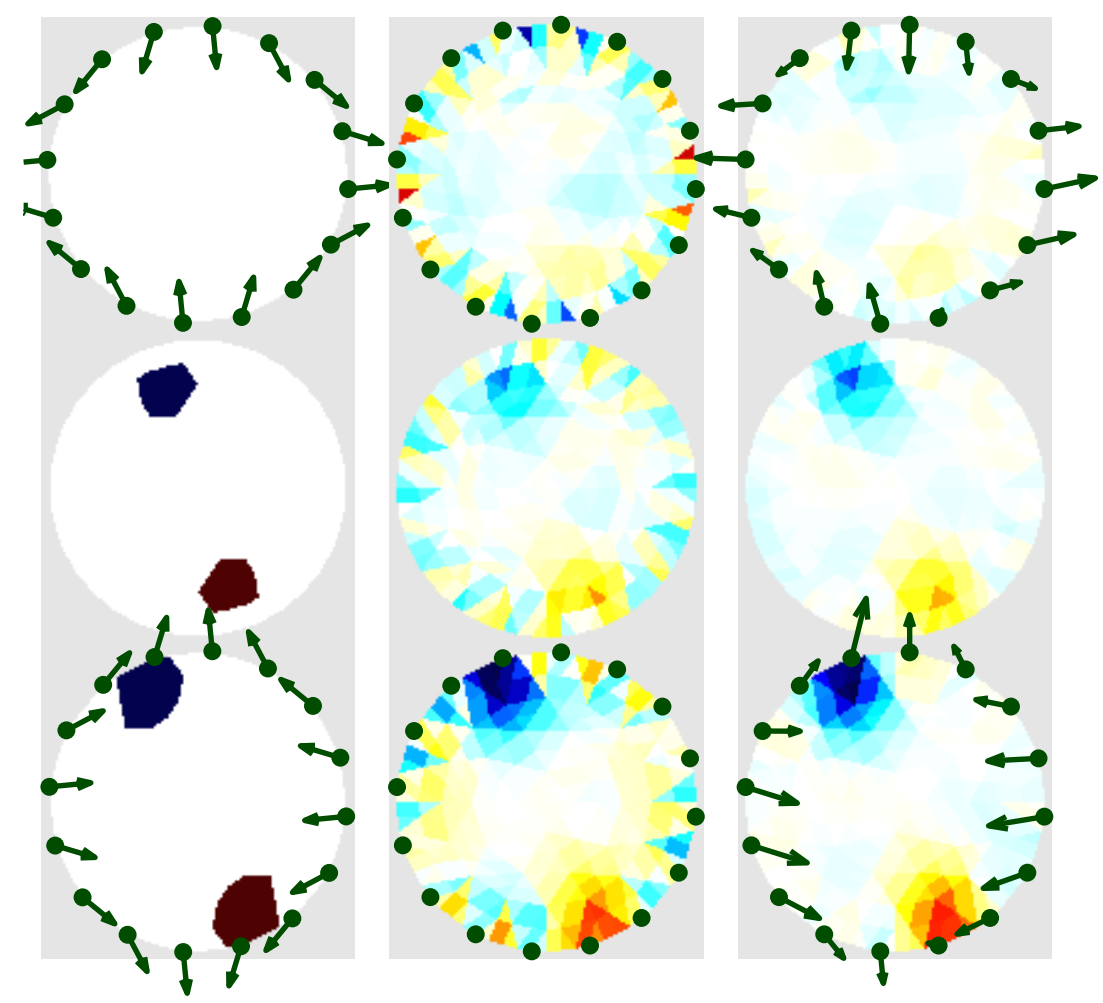

Figure 3. Reconstructed images and electrode movement from simulated 3D data with $20 \mathrm{~dB}$ SNR noise, using hyperparameters $\lambda=3 \times 10^{-3}$ and $\mu=20$. Each column shows three horizontal slices of the reconstructed image (top: $z=0.167$; middle: $z=0.500$; bottom: $z=0.833$ ). Arrows indicate each electrode's movement, and are scaled by $10 \times$. Left: Simulated inhomogeneities and electrode movements. Middle: Reconstructed image using the standard algorithm $(\mathrm{AAM}=0.0708)$. Right: Reconstructed image using the proposed algorithm $(\mathrm{AAM}=0.0190)$.

tivity and movement priors. It is also capable of calculating electrode movements during measurement and has demonstrated these results in $2 \mathrm{D}$ and $3 \mathrm{D}$ simulation. As such, compensation for boundary motion and calculation of electrode position uncertainty could make a significant improvement of in vivo EIT imaging accuracy.

\section{Acknowledgments}

This work was supported by a grant from the Natural Sciences and Engineering Research Council of Canada.

\section{References}

[1] A. Adler, and R. Guardo, "Electrical impedance tomography: Regularized imaging and contrast detection," IEEE Trans. Med. Imaging, vol. 15, pp. 170-179, 1996.

[2] A. Adler, R. Guardo, and Y. Berthiaume Y, "Impedance imaging of lung ventilation: Do we need to account for chest expansion?," IEEE Trans. Biomed. Eng., vol. 43, no. 4, pp. 414-420, 1996.

[3] B.H. Blott, G.J. Daniell, and S. Meeson, "Electrical impedance tomography with compensation for electrode positioning variations," Phys. Med. Biol., vol. 43, pp. 1731-1739, 1998.
[4] W. Breckon, and M. Pidcock, "Data errors and reconstruction algorithms in electrical impedance tomography," Clin. Phys. Physiol. Meas., vol. 9, suppl. A, pp. 105-109, 1988.

[5] V. Kolehmainen, M. Vauhkonen, P.A. Karjalainen, and J.P. Kaipio, "Assessment of errors in static electrical impedance tomography with adjacent and trigonometric current patterns," Physiol. Meas., vol. 18, pp. 289-303, 1997.

[6] V. Kolehmainen, M. Lassas, and P. Ola, "Inverse conductivity problem with an imperfectly known boundary," SIAM J. Appl. Math., vol. 66, no. 2, pp. 365-383, 2006.

[7] A. Lozano, J. Rosell, and R. Pallás-Areny, "Errors in prolonged electrical impedance measurements due to electrode repositioning and postural changes," Physiol. Meas., vol. 16, pp. 121-130, 1995.

[8] M. Soleimani, J.F.P.J. Abascal, and W.R.B. Lionheart, "Simultaneous reconstruction of the boundary shape and conductivity in 3D electrical impedance tomography," Proc. XII. Int. Conf. on Electrical Bio-Impedance V. Electrical Impedance Tomography, Gdansk, Poland, pp. 475-478, 2004. 